\title{
Simulação hidrológica ferramenta para gestão dos recursos hídricos em função de mudanças climáticas na bacia do Rio Riachão, MG, Brasil
}

\author{
Rafael Alexandre SÁ ${ }^{1}$, Marcos Koiti KONDO ${ }^{2}$, Edson de Oliveira VIEIRA ${ }^{1}$, \\ Silvânio Rodrigues dos SANTOS ${ }^{2}$, Nayara Paula Andrade VIEIRA ${ }^{3 *}$, Samuel Cola PIZETTA ${ }^{3}$
}

\author{
${ }^{1}$ Universidade Federal de Minas Gerais, Montes Claros, MG, Brasil. \\ ${ }^{2}$ Universidade Estadual de Montes Claros, Montes Claros, MG, Brasil. \\ ${ }^{3}$ Universidade Federal de Lavras, Lavras, MG, Brasil. \\ *E-mail: nayara.paula.ufla@gmail.com
}

Recebido em janeiro/2019; Aceito em julho/2019.

\begin{abstract}
RESUMO: A simulação hidrológica de bacias hidrográficas tem se tornado uma ferramenta importante de planejamento e gestão de recursos hídricos, projetando-se inclusive a disponibilidade hídrica a partir das mudanças climáticas. Dessa forma, objetivou-se avaliar a eficiência do modelo hidrológico SWAT na simulação da vazão da bacia hidrográfica do rio Riachão, no Norte de Minas Gerais, sob impacto de cenários alternativos de elevação da temperatura média do ar. O modelo SWAT foi ajustado para o período de 01/01/2008 a 31/12/2014 e calibrado com os dados das vazões hidrometradas obtendo valores do coeficiente de eficiência Nash-Sutcliffe (NSE) de 0,74 e 0,79 e tendência percentual (PBIAS) $15,45 \%$ e 16,72\%, nas fases de calibração e validação, respectivamente. A disponibilidade de água superficial da bacia hidrográfica para comparação dos cenários foi calculada por meio da curva de permanência da vazão de referência $Q_{90}$, obtendo-se o valor de $0,081 \mathrm{~m}^{3} \mathrm{~s}^{-1}$ para o modelo calibrado. Os cenários de aumento da temperatura média da bacia em 1,$5 ; 2,0 ; 3,0 ; 4,0$ e $5,0^{\circ} \mathrm{C}$ levaram ao decréscimo da Q90 em 7,66; 8,98; 10,49; 14,06 e 17,76\%, respectivamente.
\end{abstract}

Palavras-chave: escoamento superficial; modelo SWAT; cenários climáticos; gerenciamento de recursos hídricos.

\section{Hydrological simulation tool for management of water resources in the function of climate change in the Riachão river basin, $\mathrm{MG}$, Brazil}

\begin{abstract}
The hydrological simulation of watersheds becomes a major tool for planning and management of water resources, including water availability prediction from global climate change. Thus, the objective was to evaluate the efficiency of the SWAT hydrologic model to simulate the stream flow of Riachão river basin, North of Minas Gerais State, Brazil, under the impact of alternative scenarios with the increase in mean surface air temperature. The SWAT model was adjusted for 1/1/2008 to 12/31/2014 period and calibrated with data measurement obtaining values the Nash-Sutcliffe efficiency (NSE) of 0.74 and 0.79 and percent bias (PBIAS) of 15.45 and $16.72 \%$ was found to calibration and validation period, respectively. The surface water availability in the hydrographic basin was calculated by $\mathrm{Q}_{90}$ streamflow, with calibrated value of $0.081 \mathrm{~m}^{3} \mathrm{~s}^{-1}$. The scenarios of increase in mean air temperature $\left(1.5,2.0,3.0,4.0\right.$ and $\left.5.0^{\circ} \mathrm{C}\right)$ reduced $\mathrm{Q}_{90}$ by $7.66,8.98,10.49,14.06$ and $17.76 \%$, respectively.
\end{abstract}

Keywords: runoff; SWAT model; climate scenarios; water resource management.

\section{INTRODUÇÃO}

Diante do atual cenário de escassez de disponibilidade de água doce, a modelagem hidrológica de alta resolução com base física é indispensável para avaliar de forma precisa os impactos da variabilidade climática e das atividades humanas na disponibilidade de recursos hídricos (WADA et al., 2016). Nesse contexto, o SWAT (Soil and Water Assessement Tool) é um dos modelos de maior aplicabilidade e abrangência na área da hidrologia (FRANCESCONI et al., 2016; GOLMOHAMMADI et al., 2017; MALAGÒ et al., 2016).

Esse modelo é frequentemente utilizado para estimar o fluxo de nutrientes e sedimentos de uma bacia hidrográfica, atuando dessa forma como uma ferramenta de planejamento regional (GASSMAN et al., 2014). Vigiak et al. (2017) complementam que o modelo SWAT permite contabilizar várias ações de gerenciamento, tais como: práticas de manejo do solo e monitoramento de reservatórios.
As características físicas do solo e do clima suprem o modelo com os parâmetros físico-hídricos, as variáveis climáticas de entrada, e as calibrações desses parâmetros ajustam o modelo para a finalidade desejada - simulação de vazão ou hidrossedimentos (VILAYSANE et al., 2015).

Recentemente, alguns estudos têm avaliado os impactos das mudanças climáticas nas variações hidrológicas de algumas bacias hidrográficas, dentre eles pode-se destacar, Ficklin et al. (2013) no Lago Mono - Califórnia, Estados Unidos, Oliveira et al. (2017) no Rio Grande, Sudeste do Brasil e Xu et al. (2013) no Rio Qiantang, localizado no Leste da China. Estes estudos relatam que a mudança climática pode impactar substancialmente o ciclo hidrológico.

Esses impactos foram detalhados no relatório do IPCC (2014) para a América do Sul, com destaque para a redução da umidade média dos solos, alterações nos padrões de precipitação principalmente nos fatores de variabilidade e 
intensidade, o que afetaria diretamente a disponibilidade e distribuição temporal das vazões superficiais (ANA, 2016). Alguns estudos em escala de bacias hidrográficas alinham-se com essas projeções globais, destaca-se Nóbrega et al. (2011) na bacia do Rio Grande, sudeste do Brasil e Dalagnol et al. (2017) na bacia do Rio Purus, sudoeste da Amazônia.

A bacia hidrográfica do rio Riachão encontra-se em situação de conflito hídrico declarado pelo Conselho Estadual de Recursos Hídricos desde 2005, possivelmente devido ao uso intensivo da água para a agricultura, modificações antrópicas do uso e cobertura do solo e às alterações climáticas refletidas no regime de chuvas e na temperatura média do ar.

Esses fatores hidroambientais contribuem para a redução do escoamento superficial na calha do rio Riachão e, por consequência, leva ao aumento da exploração das águas subterrâneas com a instalação de poços tubulares às margens do rio, dificultando o acesso do agricultor ribeirinho ao recurso hídrico (VELOSO et al., 2011).

Nessa perspectiva, objetivou-se avaliar a eficiência do modelo hidrológico SWAT na simulação das vazões da bacia hidrográfica do rio Riachão sob impacto de cenários alternativos da elevação da temperatura do ar em 1,5;2;3; 4 e $5^{\circ} \mathrm{C}$ e seus efeitos sobre o planejamento e gestão das águas.

\section{MATERIAL E MÉTODOS}

2.1 Região de estudo e banco de dados

A bacia do rio Riachão está localizada na mesorregião do Norte de Minas Gerais e na microrregião geográfica de Montes Claros - MG, com área aproximada de $1.130 \mathrm{~km}^{2}$. O clima, conforme a classificação de Köppen é do tipo Aw tropical com inverno seco (IGAM, 2010; ALVARES et al., 2013).

O Instituto Nacional de Meteorologia - INMET forneceu os dados diários de monitoramento climático (temperaturas máximas e mínimas do ar, radiação solar, velocidade do vento e umidade relativa). Os dados de precipitação foram obtidos do Sistema de Informações Hidrológicas (HidroWeb), da Agência Nacional de Águas - ANA (Tabela 1).

Tabela 1. Código das estações climatológicas do INMET e das estações pluviométricas da ANA.

Table 1. INMET and Rainfall Stations of ANA weather stations' codes.

\begin{tabular}{ccc}
\hline Estação & Nome & Código \\
\hline Meteorológicas & Montes Claros & 83437 \\
Meteorológicas & Januária & 83386 \\
Meteorológicas & Arinos & 83384 \\
Pluviométrica & Alvação & 1644032 \\
Pluviométrica & Muquém & 1644042 \\
Pluviométrica & Nova Esperança & 1643037 \\
\hline
\end{tabular}

Os dados de vazão foram obtidos da estação fluviométrica operada pelo Instituto Mineiro de Gestão das Águas - IGAM, denominada estação Fernão Dias - Fiscal 14, localizada próxima à foz do rio Riachão, nas coordenadas: $16^{\circ} 24^{\prime} 13,2^{\prime \prime} \mathrm{S}$ e 442 25'34,0" O (WGS 84) (Figura 1A).

A bacia hidrográfica foi delimitada pelo Modelo Digital de Elevação - MDE, obtido do Banco de Dados Geomorfométricos do Brasil - TOPODATA do Instituto Nacional de Pesquisas Espaciais - INPE. O mapa de declividade (Figura 1B) foi obtido a partir de ferramentas do ArcGis 10.1 .
Para determinação das classes de solo, os pontos de coleta foram baseados no Mapa de Solos do Estado de Minas Gerais (FERNANDES FILHO; CURI, 2010) na escala de 1:500.000 (Figura 1C). Os atributos físicos foram determinados conforme Empresa Brasileira de Pesquisa Agropecuárias EMBRAPA (1997) e a erodibilidade conforme Arraes et al. (2010). O mapa de uso e ocupação do solo (Figura 1D) foi feito com base na cena de 15/09/2008, correspondente ao fim da estação seca na região, processando-se a imagem capturada pelo satélite Landsat $5 \mathrm{TM}$ (Thematic Mapper), disponibilizada pelo INPE.

\subsection{Simulação hidrológica}

Para determinação do balanço hídrico, o modelo SWAT considera quatro volumes: os reservatórios superficial, sub superficial e subterrâneo, raso e profundo, representados na Equação 1 (ABBASPOUR, 2015).

$S W_{t}=S W_{0}+\sum_{i=1}^{t}\left(R_{d a y}-Q_{\text {surf }}-E_{a}-w_{\text {seep }}-Q_{g w}\right)$

em que: $\mathrm{SWt}=$ quantidade final de água no solo $(\mathrm{mm} \mathrm{H} 2 \mathrm{O}) ; \mathrm{SW} 0=$ umidade do solo inicial no dia (i) (mm H2O); $\mathrm{t}=$ tempo (dias); Rday $=$ quantidade de precipitação no dia (i) $(\mathrm{mm} \mathrm{H} 2 \mathrm{O})$; Qsurf = quantidade de escoamento superficial no dia (i) $(\mathrm{mm} \mathrm{H} 2 \mathrm{O})$; $\mathrm{Ea} \neg=$ quantidade de evapotranspiração no dia (i) $(\mathrm{mm} \mathrm{H} 2 \mathrm{O})$; wseep = quantidade de água que entra na zona de aeração do perfil do solo no dia (i) $(\mathrm{mm} \mathrm{H} 2 \mathrm{O})$; Qgw = quantidade de retorno do fluxo no dia (i) (mm H2O).

Em Rodrigues (2017) encontra-se uma descrição mais detalhada de como se quantificam cada parâmetro da equação 1. Após a entrada de dados no modelo e execução da primeira modelagem, foram identificados os parâmetros mais influentes, para que pudesse ser feita a calibração destes.

Modificando-se os valores dos parâmetros e avaliando parte da série histórica de monitoramento das vazões (01/01/2008 a 31/12/2010), realizou-se a calibração do modelo hidrológico. Para validação desse ajuste, aplicaram-se os mesmos valores dos parâmetros calibrados no período de 01/01/2011 a 31/12/2014 (SANTOS; FONTES, 2014). Os valores de entrada correspondentes ao ano de 2008 foram utilizados como aquecimento do modelo para eliminar as incertezas devido ao desconhecimento das condições iniciais, principalmente da umidade do solo e do crescimento vegetativo, conforme apresentado por Aragão et al. (2013). Para avaliar o desempenho do modelo nas fases de calibração e validação, utilizou-se o coeficiente de Nash-Sutcliffe - NSE (Equação 2) e tendência percentual - PBIAS (Equação 3):

$N S E=1-\left(\frac{\sum_{i=1}^{n}\left(E_{m}-E_{S}\right)^{2}}{\sum_{i=1}^{n}\left(E_{m}-\bar{E}_{m}\right)^{2}}\right)$
$P B I A S=\left[\frac{\sum_{i=1}^{n} E_{i}{ }^{m}-E_{i}{ }^{s} x(100)}{\sum_{i=1}^{n}\left(E_{i}{ }^{m}\right)}\right]$

em que: Em e Em são os eventos observados e Es e Es são os eventos simulados.

O NSE pode variar de negativo infinito a 1 , no qual 1 representa um perfeito ajuste. $\mathrm{O}$ resultado da equação indica um desempenho satisfatório se estiver entre 0,7 e 0,85 . O PBIAS indica o desvio dos dados simulados em relação aos observados, com desempenho satisfatório entre 15 e $25 \%$ (SILVA; MEDEIROS, 2014). 
Nativa, Sinop, v. 7, n. 6, p. 718-726, nov./dez. 2019.

Pesquisas Agrárias e Ambientais

DOl: http://dx.doi.org/10.31413/nativa.v7i6.7803

http://www.ufmt.br/nativa

ISSN: 2318-7670
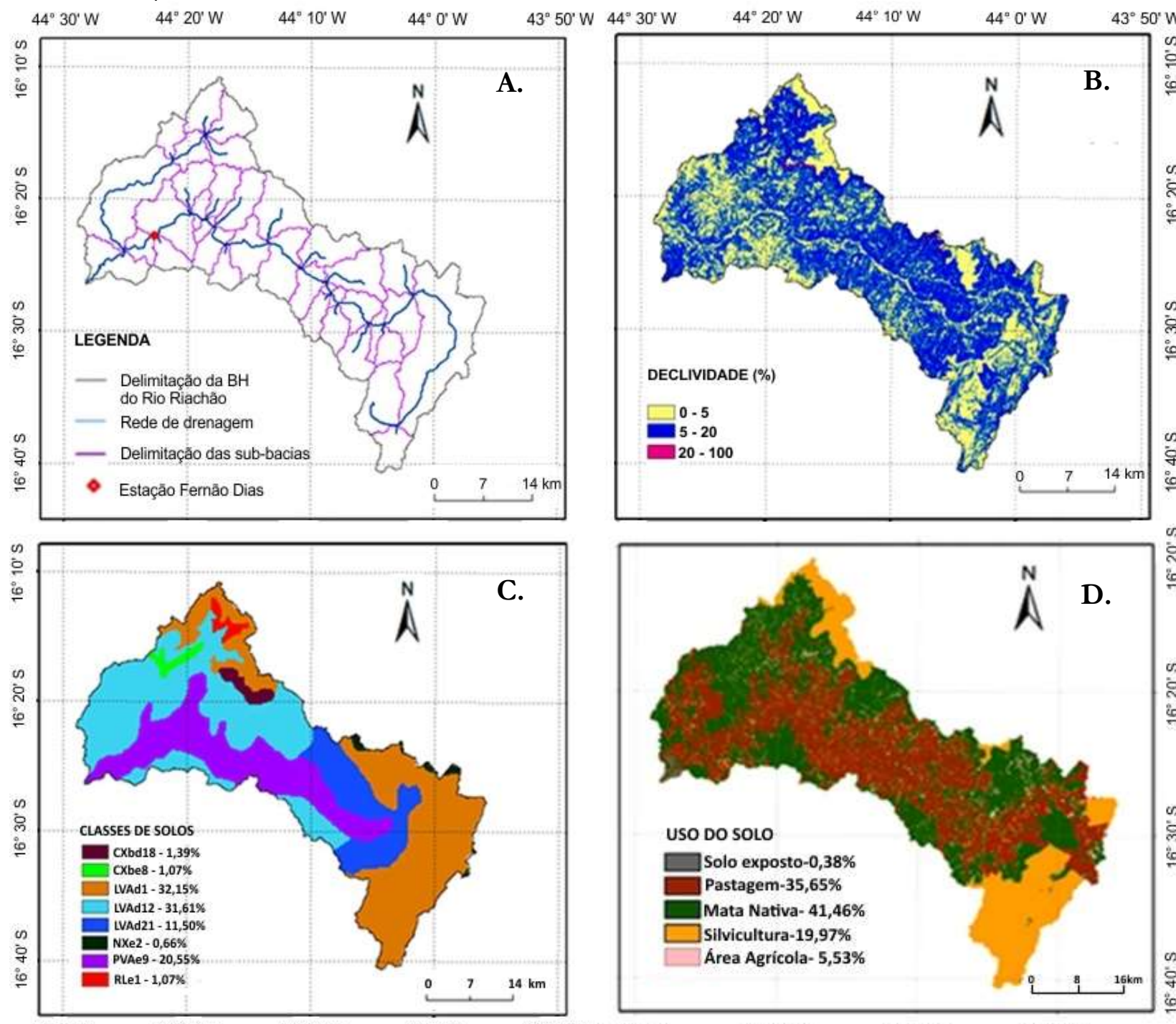

$44^{\circ} 30^{\prime} \mathrm{W} \quad 44^{\circ} 20^{\circ} \mathrm{W} \quad 44^{\circ} 10^{\prime} \mathrm{W} \quad 44^{\circ} 0^{\circ} \mathrm{W} \quad 43^{\circ} 50^{\circ} \mathrm{W} 44^{\circ} 30^{\circ} \mathrm{W} \quad 44^{\circ} 20^{\circ} \mathrm{W} \quad 44^{\circ} 10^{\circ} \mathrm{W} \quad 44^{\circ} 0^{\circ} \mathrm{W}$

Figura 1. Mapa de delimitação da bacia, sub-bacias e rede de drenagem (A.); mapa de declividades (B.); mapa de solos (C.); e mapa de uso do solo (D.) da bacia hidrográfica do rio Riachão.

Figure 1. Maps of delineation of the basin, sub-basins and drainage network (A.); slopes (B.); soils (C.); and land use (D.) of the Riachão river basin.

\subsection{Cenários estudados}

Para a avaliação dos impactos da elevação da temperatura média do ar, na disponibilidade de água da bacia hidrográfica do rio Riachão foram construídos cinco cenários de elevação da temperatura atmosférica com base no quinto relatório do Painel Intergovernamental sobre Mudanças Climáticas - IPCC (IPCC, 2014). Neste relatório estima-se impactos na disponibilidade hídrica em cenários de aumento ente $1^{\circ} \mathrm{C}$ e 4 ${ }^{\circ} \mathrm{C}$.

Baseando-se nessas projeções, foram analisados cinco cenários com elevação da temperatura média do ar em $1,5^{\circ} \mathrm{C}$; $2^{\circ} \mathrm{C} ; 3^{\circ} \mathrm{C} ; 4^{\circ} \mathrm{C}$ e $5^{\circ} \mathrm{C}$, sendo avaliados os impactos na disponibilidade de água na bacia do rio Riachão por meio da

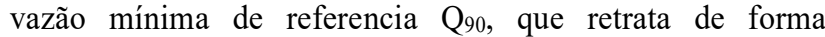
estatística a curva de permanência entre a vazão e a frequência com que essa vazão é igualada ou superada em $90 \%$ do tempo de avaliação.
2.4. Demanda hídrica em face ao uso e ocupação do solo e os impactos dos cenários sobre o percentual de atendimento aos usos econômicos identificados

A bacia hidrográfica do rio Riachão, segundo o mapa temático apresentado na (Figura 1D) tem como área agrícola $5,53 \%$ da área relativa da bacia, o que corresponde a 6.248,9 ha.

Como anteriormente apresentado, a imagem utilizada na elaboração do mapa temático "Uso e Ocupação do Solo" refere-se à data de setembro de 2008, sendo que as áreas agrícolas reconhecidas se tratam uma reflectância sensorial de áreas agrícolas irrigadas. Para a pastagem foi calculada uma área de 40.184,5 ha, o que corresponde a $35,65 \%$ da área relativa à bacia. Sendo esses os principais usos econômicos identificados.

Segundo o Manual de Outorga Estado de Minas Gerais (IGAM, 2010) a avaliação de demanda de consumo em vazão instantânea para irrigação, quando da análise de processo de 
outorga, em um sistema convencional de aspersão é de $11 \mathrm{~s}^{-1}$ ha ${ }^{-1}$, enquanto a demanda média por bovinos é de $601 \mathrm{dia}^{-1}$.

A lotação média em fazendas na região de Montes Claros - MG, segundo Barbosa (2008) em regime extensivo é de 0,7 unidade por hectare. Esses parâmetros foram utilizados para mensuração da demanda de recursos hídricos na bacia hidrográfica do rio Riachão.

Para levantamento dos usos na bacia do rio Riachão foi utilizado o banco de dados do cadastro usuários de recursos hídricos superficiais da bacia, realizado em 2005-2006 pelo IGAM, esse cadastro permite calcular a quantidade pontos de captações cadastrados, no rio Riachão e nos seus afluentes (Tabela 2).

Tabela 2. Cadastro de usuários de água superficial na bacia hidrográfica do rio Riachão - 2005/2006.

Table 4. Register of surface water users of the Riachão river basin 2005/2006.

\begin{tabular}{cccc}
\hline \multirow{2}{*}{$\begin{array}{c}\text { Banco de } \\
\text { dados }\end{array}$} & $\begin{array}{c}\text { Pontos de } \\
\text { captação }\end{array}$ & $\begin{array}{c}\text { Agricultura } \\
\text { irrigada }\end{array}$ & $\begin{array}{c}\text { Dessedentação } \\
\text { animal }\end{array}$ \\
\cline { 3 - 4 } & Cadastro & 488 & \multicolumn{2}{c}{$\left(\mathrm{m}^{3} \mathrm{~s}^{-1}\right)$} \\
\hline
\end{tabular}

Fonte: IGAM, 2010.

O banco de dados do Sistema Integrado de Informações Ambientais do Estado de Minas Gerais não possui informações representativas de usuários regularizados na bacia, sendo que suas informações não foram computadas nesse cálculo.

Por meio do cálculo dos parâmetros estabelecidos pelo Manual de Outorga de Minas Gerais (IGAM, 2010) e, pelo o apresentado por Barbosa (2008) referente à pecuária extensiva, e em função da área mapeada foi possível estimar a demanda dos usuários de água na bacia e o atendimento superficial e subterrâneo a essa demanda

\section{RESULTADOS}

3.1. Simulação hidrológica na bacia do rio Riachão

A análise visual do hidrograma, elaborado com a simulação de vazão diária e a série diária pluviométrica da bacia, apresenta-se como uma importante ferramenta para compreensão do comportamento dos processos hidrológicos e direciona os primeiros ajustes de calibração.

$\mathrm{Na}$ Tabela 2 estão listados os parâmetros calibrados pelo modelo SWAT.

Tabela 2. Parâmetros utilizados na calibração do modelo SWAT na bacia do rio Riachão.

Table 2. Parameters used in the calibration of the SWAT model of the Riachão river basin.

\begin{tabular}{cccc}
\hline Parâmetro & $\begin{array}{c}\text { Intervalo do } \\
\text { parâmetro }\end{array}$ & $\begin{array}{c}\text { Valor } \\
\text { modelado }\end{array}$ & $\begin{array}{c}\text { Valor } \\
\text { calibrado }\end{array}$ \\
\hline ALPHA_BF & 0 a 1 & 0,048 & 0,05 \\
CH_K & $-0,01$ a500 & 0 & 15 \\
EPCO & 0 a 1 & 1 & 0,12 \\
GW_DERLAY & 0 a 500 & 31 & 10 \\
SURLAG & 0 a 24 & 2 & 1 \\
\hline
\end{tabular}

Os hidrogramas das vazões diárias simuladas e hidrometradas no período de calibração e validação estão ilustrados nas Figuras 2A e 2B, respectivamente.

Os valores do coeficiente Nash-Sutcliffe (NSE) foram 0,74 e 0,79 nas fases de calibração e validação, respectivamente. $\mathrm{Na}$ avaliação da estatística de precisão
PBIAS obtiveram-se os valores de $15,45 \%$ na fase de calibração e $16,72 \%$ na validação.

Baseado nos resultados obtidos na calibração do modelo pode-se inferir que o SWAT é apto para simular de forma satisfatória os processos hidrológicos na bacia do rio Riachão.
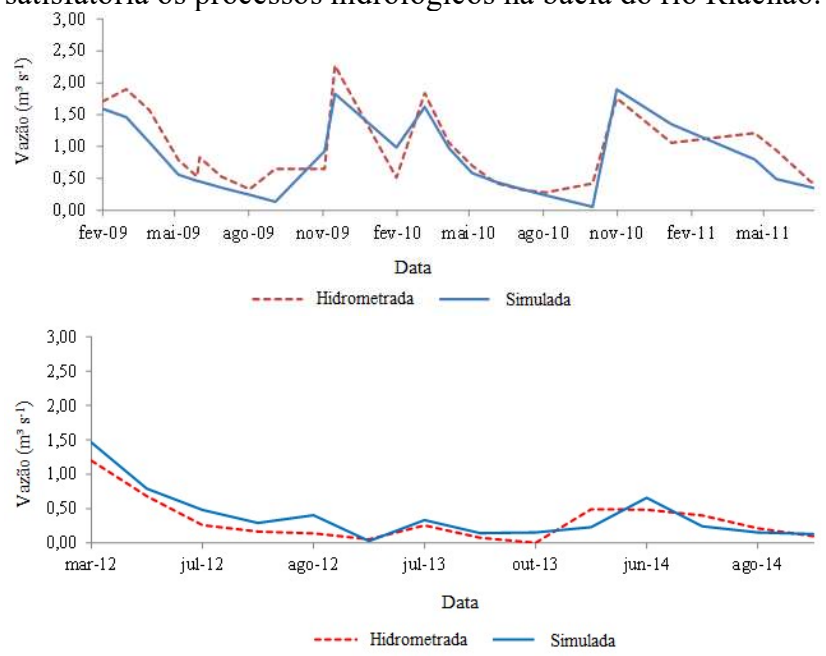

Figura 2. Hidrograma hidrometrado e simulado para a bacia do rio Riachão no período de calibração (A.) e validação (B.).

Figure 2. Observed and simulated hydrogram for the Riachão river basin during the calibration (A.) and validation (B.) period.

3.2. Mensuração da disponibilidade de água na bacia hidrográfica e avaliação dos impactos dos cenários de elevação da temperatura média do ar sobre a disponibilidade de água

A disponibilidade de água em uma bacia hidrográfica foi vinculada à vazão de referência, com garantia de permanência durante o ano, que passa a representar o limite máximo de utilização da água. Na determinação da vazão de referência $\mathrm{Q}_{90}$ as vazões diárias simuladas com os parâmetros calibrados, foram divididas em 11 classes com amplitude de $0,227 \mathrm{~m}^{3} \mathrm{~s}^{-1}$, onde foram calculadas as frequências de ocorrência dos dados em cada classe e elaborado a curva de permanência de vazões no rio Riachão (Figura 3).

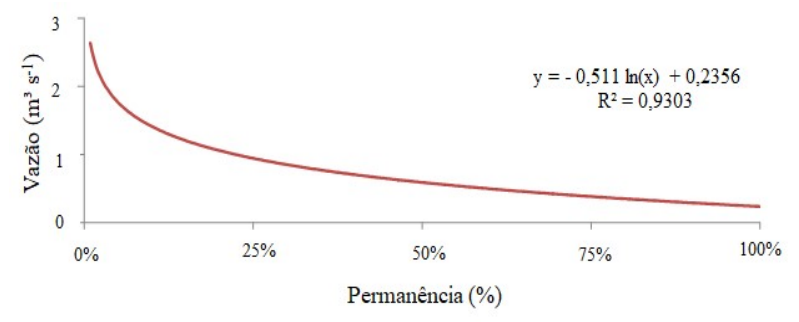

Figura 3. Curva de permanência de vazões no rio Riachão.

Figure 3. Permanence of flows curve on the Riachão river.

$\mathrm{Na}$ avaliação dos impactos dos cenários de elevação da temperatura média do ar sobre a disponibilidade de água fixou os valores de parâmetros calibrados, alterou-se nas tabelas de entrada a temperatura máxima e mínima diária, obtidas por meio das estações meteorológicas (Tabela 1), acrescentandose a elas os valores da elevação de temperatura projetados nos cenários.

Essas alterações de temperatura foram utilizadas para simulação dos cenários na bacia. Os resultados foram mensurados e avaliados por novos cálculos do valor da $\mathrm{Q}_{90}$ tendo como referência os demais parâmetros do modelo calibrado e os cenários de elevação da temperatura. 
Com a variação da temperatura atmosférica houve uma modificação dos valores de disponibilidade hídrica superficial na bacia do rio Riachão, essa modificação se refletiu em um decréscimo dos valores correspondentes à $\mathrm{Q}_{90}$.

$\mathrm{Na}$ Figura 4 está ilustrada a frequência de vazões distribuídas em classes para os cenários simulados.

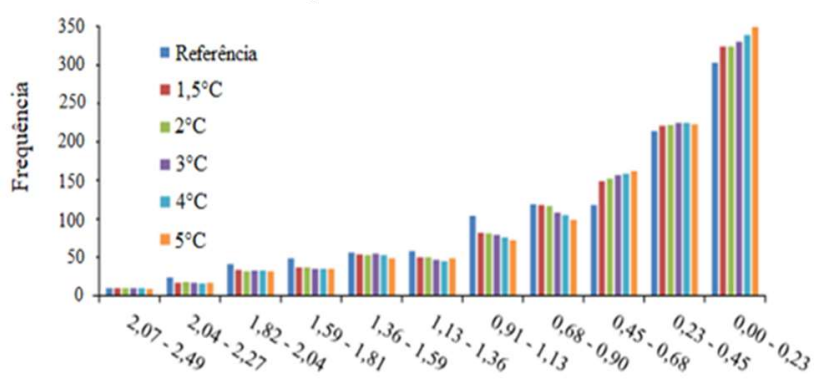

Classe de vazões $\left(\mathrm{m}^{3} \mathrm{~s}^{-1}\right)$

Figura 4. Frequência de vazões distribuídas em 11 classes para cenários simulados.

Figure 4. Frequency of flows distributed in 11 classes for simulated scenarios.

Para melhor ilustrar esse impacto sobre as vazões mínimas, a $Q_{90}$ foi descrita para cada cenário e plotada no gráfico para visualização do decréscimo da disponibilidade de recurso hídrico superficial na bacia do rio Riachão (Figura 5).

Com base nesses resultados foi possível determinar o impacto da alteração da temperatura média do ar sobre a disponibilidade de água na bacia do rio Riachão (Tabela 3), onde se verificou um decréscimo de 7,66, 8,98, 10,49, 14,06, $17,76 \%$, respectivamente.

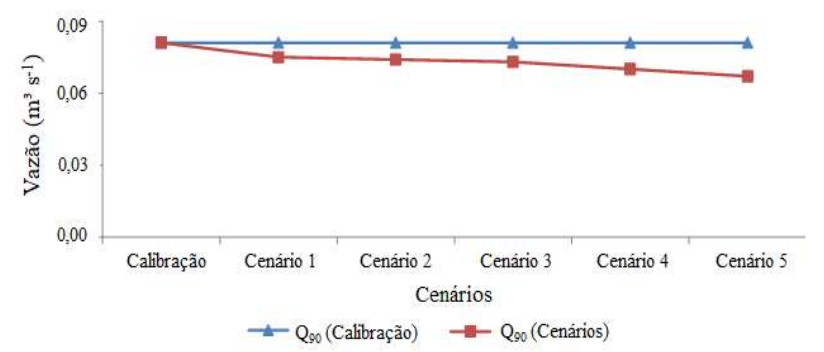

Figura 5. Cenários avaliados e valores correspondentes da vazão Q90 na bacia hidrográfica do rio Riachão.

Figure 5. Evaluated scenarios and corresponding values of the $\mathrm{Q}_{90}$ of the Riachão river basin.

Tabela 3. Valores da vazão de referência $Q_{90}$ e o percentual de decréscimo em dois cenários de mudanças climáticas.

Table 3. Q90 flow values and percentage decrease in two climate change scenarios.

\begin{tabular}{|c|c|c|c|}
\hline $\begin{array}{l}\text { Parâmetros } \\
\text { climáticos }\end{array}$ & \multicolumn{2}{|c|}{$\mathrm{Q}_{90}\left(\mathrm{~m}^{3} / \mathrm{s}\right)$} & Decréscimo (\%) \\
\hline Calibração & *T observada & 0,081 & Valor de referência \\
\hline Cenário 1 & $* \mathrm{~T}+1,5^{\circ} \mathrm{C}$ & 0,075 & $-7,66$ \\
\hline Cenário 2 & $* \mathrm{~T}+2,0^{\circ} \mathrm{C}$ & 0,074 & $-8,98$ \\
\hline Cenário 3 & $* \mathrm{~T}+3,0^{\circ} \mathrm{C}$ & 0,073 & $-10,49$ \\
\hline Cenário 4 & $* \mathrm{~T}+4,0^{\circ} \mathrm{C}$ & 0,070 & $-14,06$ \\
\hline Cenário 5 & $* \mathrm{~T}+5,0^{\circ} \mathrm{C}$ & 0,067 & $-17,76$ \\
\hline
\end{tabular}

*T: temperatura

3.4. Demanda hídrica em face ao uso e ocupação do solo e os impactos dos cenários sobre o percentual de atendimento aos usos econômicos identificados
$\mathrm{Na}$ Tabela 5 encontra-se a estimativa da demanda dos usuários de água na bacia e o percentual de atendimento superficial e subterrâneo a essa demanda.

Dentro desse percentual de atendimento na bacia hidrográfica foram projetados os cenários de simulação de aumento da temperatura e reduções de rendimento na curva de permanência, considerando que com a diminuição da disponibilidade de água superficial haverá uma compensação pelo aumento da explotação de água subterrânea. Nesse contexto apresenta-se a seguinte situação (Tabela 6).

Tabela 5. Percentual de atendimento a demanda de recursos hídricos na bacia hidrográfica do rio Riachão entre captações superficiais e subterrâneas.

Table 5. Percentage of meeting the demand of water resources of the Riachão river basin between surface and ground abstractions.

\begin{tabular}{|c|c|c|c|}
\hline \multirow{2}{*}{$\begin{array}{c}\text { Tipo de } \\
\text { Captação }\end{array}$} & $\begin{array}{l}\text { Agricultura } \\
\text { irrigada }\end{array}$ & $\begin{array}{l}\text { Dessedentação } \\
\text { animal }\end{array}$ & \multirow[t]{2}{*}{$\begin{array}{l}\text { Percentual de } \\
\text { atendimento }\end{array}$} \\
\hline & \multicolumn{2}{|c|}{$\left(\mathrm{m}^{3} \mathrm{~s}^{-1}\right)$} & \\
\hline Superficial & 1,211 & 0,005 & $19,40 \%$ \\
\hline Subterrânea & 5,038 & 0,014 & $80,60 \%$ \\
\hline
\end{tabular}

Tabela 6. Percentual de atendimento às demandas de recursos hídricos pelo fluxo superficial e reservas subterrâneas e transferência desse atendimento conforme os cenários de aumento da temperatura média do ar.

Table 6. Percentage of service to the demands of water resources by surface flow and underground reserves and transfer of this service according to the scenarios of increase of average air temperature. Cenários com redução percentual Q90

\begin{tabular}{cccccc} 
& \multicolumn{5}{c}{$(\%)$} \\
\cline { 2 - 6 } Tipo de Captação & $\begin{array}{c}(1)- \\
7,66\end{array}$ & 8,98 & $\begin{array}{c}(3)- \\
10,49\end{array}$ & $\begin{array}{c}(4)- \\
14,06\end{array}$ & $\begin{array}{c}(5)- \\
17,76\end{array}$ \\
\hline $\begin{array}{c}\text { Percentual de } \\
\text { atendimento } \\
\text { Superficial (\%) }\end{array}$ & 17,91 & 17,66 & 17,36 & 16,67 & 15,45 \\
\hline $\begin{array}{c}\text { Percentual de } \\
\text { atendimento } \\
\text { subterrâneo (\%) }\end{array}$ & 82,09 & 82,34 & 82,64 & 83,33 & 84,55 \\
\hline $\begin{array}{c}\text { Transferência de } \\
\text { atendimento em (m } \\
\left.\mathrm{s}^{-1}\right)\end{array}$ & 0,093 & 0,110 & 0,128 & 0,171 & 0,216 \\
\hline
\end{tabular}

Nota-se que quanto menor a disponibilidade de água superficial na bacia hidrográfica do rio Riachão, menor será o percentual de atendimento dessa fonte de recursos hídricos à demanda de uso da bacia. Impera destacar que mesmo no cenário mais favorável (cenário 1) a transferência do atendimento supera o valor de $\mathrm{Q}_{90}$ calculado na estação Fernão Dias, ao avançar na severidade dos cenários esses valores elevam-se conforme apresentado na Tabela 6.

\section{DISCUSSÃO}

\subsection{Simulação hidrológica na bacia do rio Riachão}

As vazões mínimas no período de recessão na bacia se mantiveram permanentes com valores abaixo de $1 \mathrm{~m}^{3} \mathrm{~s}^{-1}$, adequados aos valores hidrometrados (Figuras 2A e 2B). De acordo com Alexandre Sá (2016) há registros de baixas vazões no período de recessão no curso de água do rio Riachão sem histórico de intermitência na estação Fernão Dias. Porém, na porção alto-média da bacia se apresenta histórico de intermitência pelo uso excessivo de água para a agropecuária e verifica-se a presença de sumidouros e surgências em trechos do leito principal, característicos da formação geológica calcária e carstificada bacia (SOARES; VELÁSQUEZ, 2013). 
Os resultados do desempenho do modelo permitem o enquadrar como "muito bom" em ambas as fases para o NSE e satisfatório para o PBIAS, nessa situação, adota-se a avaliação mais conservadora, indicando um ajuste satisfatório do modelo (PEREIRA et al., 2014).

De forma similar ao presente trabalho, Devkota; Gyawali (2015) e Eduardo et al. (2016) utilizaram as estatísticas de precisão NSE e o PBIAS para testar a eficiência de modelos hidrológicos em bacias hidrográficas. Devkota; Gyawali (2015) encontravam valores de NSE de 0,97 e 0,87 e PBIAS de $0,21 \%$ e $6,5 \%$ nas fases de calibração e validação respectivamente. Os autores concluíram que o modelo SWAT foi capaz de reproduzir a hidrologia fluvial do rio Koshi, Nepal. Eduardo et al. (2016) encontraram valores de NSE de 0,76 e 0,62 para os períodos de calibração e validação, respectivamente, o que fornece a base para o modelo ser classificado como bom e muito bom na simulação hidrológica da bacia hidrográfica do rio das Mortes, MG.

4.2. Mensuração da disponibilidade de água na bacia hidrográfica e avaliação dos impactos dos cenários de elevação da temperatura média do ar sobre a disponibilidade de água

Pode-se verificar na Tabela 6 que as variações entre as frequências de vazões por cenários ocorreram mais intensamente nas classes de menores vazões. $\mathrm{Na}$ classe de menor vazão $\left(0,00\right.$ a $\left.0,23 \mathrm{~m}^{3} \mathrm{~s}^{-1}\right)$, correspondente à vazão de permanência $Q_{90}$, tornou-se mais evidente essa variação. Tal fato mostra que a elevação da temperatura afeta em maior intensidade às vazões mínimas do curso de água Riachão o que afeta diretamente a disponibilidade de água superficial na bacia.

Os resultados de decréscimo na $\mathrm{Q}_{90}$ são esperados tendo em vista que a temperatura é uma variável que integra o cálculo da evapotranspiração, fator de saída de água do balanço hídrico de uma bacia hidrográfica. Segundo Vieira et al. (2016) a temperatura média do ar é um dos fatores que mais influenciam o processo de evaporação.

$\mathrm{O}$ aumento da temperatura do ar, independente, da variação da precipitação ocasiona impactos diretos à disponibilidade de água na bacia hidrográfica, em razão da elevação dos índices de evaporação aos lagos, açudes, reservatórios e aumento da evapotranspiração das plantas. Se, a estes cenários de elevação de temperatura, adiciona-se a modificação do regime de chuvas e dos seus extremos, como demonstram as projeções dos modelos climáticos globais e regionais até o final do Século XXI do IPCC (2014), as atividades associadas à agricultura de subsistência podem ser bem agravadas. Nesse aspecto, o semiárido brasileiro, onde se encontra a bacia do rio Riachão, representa uma das regiões mais vulneráveis do país às mudanças e à variabilidade do clima, sobretudo aquelas associadas às secas e às enchentes (MARENGO et al., 2011).

4.3. Demanda hídrica em face do uso e ocupação do solo e os impactos dos cenários sobre o percentual de atendimento aos usos econômicos identificados

Os resultados exibidos na Tabela 5 mostram tendências semelhantes aos encontrados pelos estudos conduzidos por (Cirilo, 2008) na região do semiárido brasileiro, onde constatou-se o grande potencial dos aquíferos nordestinos em formações sedimentares, chegando a representar uma reserva renovável correspondente a $60 \%$ do reservatório de Sobradinho na Bahia.
Conforme apresentado por Euclydes et al. (2010) a vazão mínima, que reflete a disponibilidade de água na bacia do rio Riachão, pelo método $\mathrm{Q}_{7,10}$, determinada nos estudos de regionalização de vazão foi estimada em 1,22 $\mathrm{m}^{3} \mathrm{~s}^{-1}$, valor semelhante ao estimado na Tabela 5 .

Diante dos cenários verificados na Tabela 6 observam-se duas situações distintas. A primeira se refere ao atendimento das demandas de recursos hídricos na bacia quando decrescida a disponibilidade de água superficial transfere, proporcionalmente, esse déficit para as reservas subterrâneas, tal fato eleva a pressão de uso sobre essa reserva, sem que se avalie a dimensão da sustentabilidade dessa explotação no aquífero, trazendo um risco de ocorrência de um colapso dessas reservas (AMORIM et al., 2010).

A segunda reflete a situação em que com a diminuição da disponibilidade hídrica superficial na bacia, a produção agrícola e a pecuária estabilize seu crescimento fazendo que a utilização dos recursos ambientais, seja gerida com mais eficiência, ou que ocorra a retração dessa produção em face da menor disponibilidade de recursos hídricos superficiais.

Outro fato decorrente dessa simulação é que a perenidade do rio Riachão durante $o$ ano hidrológico depende sensivelmente da contribuição do fluxo de base para sua manutenção. Diante desse fato pode-se notar que a recarga dos aquíferos, assim como sua preservação tem reflexo direto na realimentação do fluxo superficial no período de recessão (REBOUÇAS, 1997).

Nesse contexto pode se afirmar que quanto mais se aumenta pressão sobre as reservas subterrâneas em função da diminuição da disponibilidade hídrica superficial, menor será essa disponibilidade, pois a contribuição responsável pelo fluxo de base passa a ser explotada, diretamente, por meio de poços profundos. Esse cenário cria um ciclo vicioso de acentuação da escassez hídrica superficial da bacia.

4.4. Consequências dos impactos projetos pelos cenários na caracterização fisiográfica da bacia do rio Riachão e possíveis alternativas de mitigação

Com a ocorrência das projeções dos cenários de elevação da temperatura média do ar espera-se a ocorrência de precipitações mais concentradas e o aumento da evaporação nos corpos d'água e por consequência a redução do fluxo de água. Segundo Cirilo (2008) projeta-se uma redução de recarga dos aquíferos em até $70 \%$ para 2050 , esse fato torna ainda mais severo o problema de escassez hídrica na bacia do rio Riachão.

Portanto, com a diminuição da realimentação da vazão do rio e a concentração do período chuvoso assevera-se a tendência de desertificação da região, com a substituição do cerrado pela caatinga, e essa por vegetação mais típica de regiões áridas, como as cactáceas (IPCC, 2014). Essa mudança de cenário de cobertura do solo traz consequências diretas à biodiversidade, fauna, flora e recursos hidroambientais.

$\mathrm{Na}$ tentativa de equilibrar esse balaço hídrico algumas alternativas devem ser levadas em consideração, principalmente aquelas relacionadas à indução de recarga dos aquíferos, que passa desde o manejo conservacionista dos sistemas de produção, por meio de estruturas de conservação do solo e da água, à infraestruturas de recargas como barragens superficiais e subterrâneas.

Essas estruturas combinadas com o uso e ocupação do solo sob critérios técnicos sustentáveis proporcionam a infiltração da água pelo solo levando ao reequilíbrio do ciclo hidrológico, 
disponibilização de água para as culturas, recarga dos aquíferos e conservação do solo e dos mananciais da bacia hidrográfica (CECÍLIO et al., 2003).

Promover a recarga dos aquíferos é um dos principais desafios de sustentabilidade na bacia hidrográfica do rio Riachão, além das técnicas de conservação de solo e água, destaca-se a importância da indução da recarga artificial dos aquíferos. Nesse sentido Rebouças (1997) ressalta que, desde 1970, as demandas de água em Israel já eram superiores aos seus potenciais renováveis. $\mathrm{O}$ déficit desde então passou a ser atendido por meio do uso intensivo das águas subterrâneas e reuso de efluentes domésticos e industriais, sendo que esses últimos eram utilizados, também, para recarga artificial de aquíferos.

Por meio da simulação dos cenários de diminuição de disponibilidade hídrica na bacia hidrográfica do rio Riachão, destaca-se o acirramento do conflito hídrico, e nesse quadro, faz-se necessário implementar um modelo de gerenciamento dos recursos hídricos eficiente resultante da consolidação das novas visões que se tornam mais evidentes nessas simulações.

A principal constatação apresentada na bacia assenta-se no fato de que os processos hidroambientais interagem diretamente com o desenvolvimento econômico/social, a biodiversidade, o uso do solo, a cobertura vegetal, sendo essas interações são sensíveis aos impactos das mudanças climáticas. Além disso, essa gestão na bacia do Riachão deve, necessariamente, integrar recursos hídricos superficiais e subterrâneos, em razão interdependência dessas reservas para a disponibilidade de água na bacia.

4.5. Impactos projetados pelos cenários na situação de conflito relacionado ao acesso à água

A escassez de água no clima semiárido apresenta-se como um problema recorrente com históricos de conflitos em bacias hidrográficas (CIRILO, 2008).

Um dos pontos de conflito insere-se quanto ao acesso à água. Em muitos casos esse acesso apresenta-se em condições diferenciadas para o agronegócio e para as comunidades rurais da agricultura familiar na bacia do rio Riachão.

$\mathrm{O}$ acesso à água, nessa conjuntura de escassez, passa a ser possível, durante os períodos críticos de estiagem, por meio de poços tubulares, estrutura de engenharia que requer investimento financeiro e técnico para aquisição.

As comunidades rurais da agricultura familiar, que estão concentradas às margens do rio Riachão e de seus afluentes, no passado, tinham acesso ao recurso hídrico superficial para atendimento de suas necessidades produtivas, fato que vem sendo modificado pela diminuição da disponibilidade de água na bacia (EUCLYDES et al., 2010). A capacidade de investimento financeiro dos diferentes setores produtivos promove à desigualdade no acesso a água na bacia do Riachão.

Todavia, apesar da agricultura familiar se mostrar como o setor mais vulnerável diante dessas variações climáticas, a capacidade de resiliência da sociedade torna-se a principal saída para enfrentamento dos cenários simulados.

Para se adaptar o gerenciamento dos recursos hídricos da bacia do rio Riachão a essa variabilidade climática deve-se observar o fato de que a avaliação dos impactos sobre a disponibilidade de água, com referência a elevação da temperatura, é um processo contínuo e o planejamento deve projetar a mobilidade dessa variável em diferentes cenários.

As ações de adaptabilidade devem ser flexibilizadas de acordo com os impactos em diferentes escalas e atores. Além disso, faz-se necessário induzir a aplicação dos avanços tecnológicos no planejamento, principalmente referentes à gestão, ao uso eficiente e às formas de acesso aos recursos hídricos, para compor a atualização de estratégias dinâmicas de gerenciamento da bacia hidrográfica do rio Riachão (OBERMAIER; ROSA, 2013).

\section{CONCLUSÕES}

O modelo SWAT é adequado para a simulação das vazões, com valores satisfatórios de coeficiente de Nash-Sutcliffe e estatística de precisão PBIAS

A elevação da temperatura média do ar reduz a vazão de referência $Q_{90}$, influenciando diretamente nas vazões mínimas.

Os cenários projetados para o futuro da bacia indicam o aumento dos conflitos pela água em função da diminuição da disponibilidade hídrica superficial na bacia e o possível avanço sobre as reservas hídricas subterrâneas, fato que assevera a escassez hídrica pelo comprometimento do fluxo de base.

O planejamento e gestão de recursos hídricos na bacia do rio Riachão devem considerar os impactos das variações climáticas no gerenciamento dos conflitos.

\section{REFERÊNCIAS}

ABBASPOUR, K. C. SWAT-CUP: SWAT Calibration and Uncertainty Programs. A User Manual. Swiss Federal Institute of Aquatic Science and Technology - EAWAG. 2015.

ALVARES, C. A.; STAPE, J. L.; SENTELHAS, P. C.; GONÇALVES, J. L. M. de; SPAROVEK, G. Köppen's climate classification map for Brazil. Meteorologische Zeitschrift, Berlin, v. 22, n. 6, p. 711-728, 2013. DOI: http://dx.doi.org/10.1127/0941-2948/2013/0507

ANA_AGÊNCIA NACIONAL DAS ÁGUAS. Mudanças Climáticas e Recursos Hídricos: avaliações e diretrizes para adaptação / Agência Nacional de Águas. Brasília: ANA, GGES, 2016. 92 p.

ARAGÃO, R. de.; CRUZ, M. A. S.; AMORIM, J. R. A. de; MENDONÇA, L. C.; FIGUEIREDO, E. E. de; SRINIVASAN, V. S. Análise de sensibilidade dos parâmetros do modelo swat e simulação dos processos hidrossedimentológicos em uma bacia no agreste Nordestino. Revista Brasileira de Ciência do Solo, Viçosa, v. 37, n. 4, p. 1091-1102, 2013. DOI: http://dx.doi.org/10.1590/S0100-06832013000400026

ARRAES, C. L.; BUENO, C. R. P.; PISSARRA, T. C. T. Estimativa da erodibilidade do solo para fins conservacionistas na microbacia córrego do Tijuco, SP. Bioscience Journal, Uberlândia, v. 26, n. 6, p. 849-857, 2010.

AMORIM, J. R. A.; CRUZ. M. A. S.; RESENDE, R. S. Qualidade da água subterrânea para irrigação na bacia hidrográfica do Rio Piauí, em Sergipe. Revista Brasileira de Engenharia Agrícola e Ambiental, Campina Grande, v. $14, \quad$ n. $8, \quad$ p. $804-811,2010$. DOI: http://dx.doi.org/10.1590/S1415-43662010000800003

BARBOSA, F. A. Viabilidade econômica de sistemas de produção de bovinos de corte em propriedades nos estados de Minas Gerais e da Bahia. 2008. 137f. Tese (Doutorado em Zootecnia) - Escola de veterinária, Universidade Federal de Minas Gerais, Belo Horizonte, 2008.

CECÍLIO, R. A.; SILVA, D. D.; PRUSKI, F. F.; MARTINEZ, M. A. Modelagem da infiltração de água no solo sob 
condições de estratificação utilizando-se a equação de Green-Ampt. Revista Brasileira de Engenharia Agrícola e Ambiental, Campina Grande, v. 7, n. 3, p. 415422, 2003. DOI: http://dx.doi.org/10.1590/S141543662003000300002

CIRILO, J. A. Políticas Públicas de recursos hídricos para o semi-árido. Estudos Avançados, São Paulo, v. 22, n. 63, p. 61-82, 2008. DOI: http://dx.doi.org/10.1590/S010340142008000200005

DALAGNOL, R.; BORMA, L. S.; MATEUS, P.; RODRIGUEZ, D. A. Assessment of climate change impacts on water resources of the Purus Basin in the southwestern Amazon. Acta Amazonica, Manaus, v. 47, n. $3, \quad$ p. 213-226, 2017. DOI: http://dx.doi.org/10.1590/1809-4392201601993

DEVKOTA, L. P.; GYAWALI, D. R. Impacts of climate change on hydrological regime and water resources management of the Koshi River Basin, Nepal. Journal of Hydrology: Regional Studies, v. 4, p. 502-515, 2015. DOI: https://dx.doi.org/10.1016/j.ejrh.2015.06.023

EDUARDO, E. N.; MELLO, C. R. de; VIOLA, M. R.; CURI, N. Hydrological simulation as subside for management of surface water resources at the Mortes River Basin. Ciência e Agrotecnologia (Online), Lavras, v. 40, n. 4, p. 390-404, 2016. DOI: http://dx.doi.org/10.1590/141370542016404009516

EMBRAPA_EMPRESA BRASILEIRA DE PESQUISA AGROPECUÁRIA. Manual de Métodos de Análise de Solo. 2. ed. Rio de Janeiro: Embrapa; CNPS, 1997. 212 p.

EUCLYDES, H. P.; FERREIRA, P. A.; FARIA FILHO, R. F.; LEITE, C. V. Impacto ambiental relevante na sub-bacia do rio Riachão, Norte de Minas Gerais: Rio Riachão palco de um conflito histórico pelo uso das suas águas. Belo Horizonte, MG: RURALMINAS, 2010.

FERNADES FILHO, E. I.; CURI, N. Mapa de solos de Minas Gerais. Belo Horizonte, MG: Fundação Estadual do Meio Ambiente (FEAM), 2010.

FICKLIN, D. L.; STEWART, I. T.; MAURER, E. P. Effects of projected climate change on the hydrology in the Mono Lake Basin, California. Climatic Change, Dordrecht, v. 116, n. 1, p. 111-131, 2013. DOI: https://doi.org/10.1007/s10584-012-0566-6

FRANCESCONI, W.; SRINIVASAN, R.; PÉREZ-MIÑANA, E.; WILLCOCK, S. P.; QUINTERO, M. Using the Soil and Water Assessment Tool (SWAT) to model ecosystem services: A systematic review. Journal of Hydrology, Amsterdam, v. 535, p. 625-636, 2016. DOI: https://dx.doi.org/10.1016/j.jhydrol.2016.01.034

GASSMAN, P. W.; SADEGHI, A. M.; SRINIVASAN, R. Applications of the SWAT model special section: overview and insights. Journal of Environmental Quality, Madison, v. 43, p. 1-8, 2014. DOI: https://dx.doi.org/10.2134/jeq2013.11.0466

GOLMOHAMMADI, G.; RUDRA, R.; DICKINSON, T.; GOEL, P.; VELIZ, M. Predicting the temporal variation of flow contributing areas using SWAT. Journal of Hydrology, Amsterdam, v. 547, p. 375-386, 2017. DOI: https://dx.doi.org/10.1016/j.jhydrol.2017.02.008

IGAM_INSTITUTO MINEIRO DE GESTÃO DAS ÁGUAS. Manual técnico e administrativo de outorga de direito de uso de recursos hídricos no estado de Minas Gerais. Belo Horizonte, MG: IGAM, 2010. 113 p.
IPCC_INTERGOVERNMENTAL PAINEL ON CLIMATE $\mathrm{C} \overline{\mathrm{H}} \mathrm{ANGE}$. Climate Change 2014: Impacts, Adaptation, and Vulnerability. Cambridge: Cambridge University Press, 2014.

MALAGÒ, A.; EFSTATHIOU, D.; BOURAOUI, F.; NIKOLAIDIS, N. P.; FRANCHINI, M.; BIDOGLIO, G.; KRITSOTAKIS, M. Regional scale hydrologic modeling of a karst-dominant geomorphology: The case study of the Island of Crete. Journal of Hydrology, Amsterdam, v. 540, p. 64-81, 2016. DOI: https://dx.doi.org/10.1016/j.jhydrol.2016.05.061

MARENGO, J. A.; NOBRE, C. A.; CHOU, S. C.; TOMASELLA, J.; SAMPAIO, G.; OBREGÓN, G. O.; SOARES, W. R.; BETTS, R.; KAY, G. Riscos das mudanças climáticas no Brasil: análise conjunta Brasil-Reino Unido sobre os impactos das mudanças climáticas e do desmatamento na Amazônia. Disponível em: <http://www.ccst.inpe.br>. Acesso em: 14 nov. 2015.

NÓBREGA, M. T.; COLLISCHONN, W.; TUCCI, C. E. M.; $\mathrm{PAZ}, \mathrm{A} . \mathrm{R}$. Uncertainty in climate change impacts on water resources in the Rio Grande Basin, Brazil. Hydrology and Earth System Sciences, v. 15, p. 585595, 2011. DOI: 10.5194/hess-15-585-2011

PEREIRA, D. dos R.; MARTINEZ, M. A.; SILVA, D. D. da; ALMEIDA, A. Q. de; PRUSKI, F. F.; SILVA, D. D. da; ZONTA, J. H. Hydrological simulation using SWAT model in headwater basin in southeast Brazil. Engenharia Agrícola, Jaboticabal, v. 34, n. 4, p.789-799, 2014. DOI: http://dx.doi.org/10.1590/S0100-69162014000400018

OBERMAIER, M.; ROSA, L. P. Mudança climática e adaptação no Brasil: uma análise crítica. Estudos Avançados, São Paulo, v. 27, n. 78, p. 155-176, 2013. DOI: $\quad$ http://dx.doi.org/10.1590/S010340142013000200011

OLIVEIRA, V. A. de; MELLO, C. R. de; VIOLA, M. R.; SRINIVASAN, R. Assessment of climate change impacts on streamflow and hydropower potential in the headwater region of the Grande river basin, Southeastern Brazil. International Journal of Climatology, Chinchester, v. 37, n. 5, p. 5005-5023, 2017. DOI: https://dx.doi.org/10.1002/joc.5138

REBOUÇAS, A. C. Água na região Nordeste: desperdício e escassez. Estudos Avançados, São Paulo, v. 11, n. 29, p. 127-154, 1997. DOI: http://dx.doi.org/10.1590/S010340141997000100007

RODRIGUES, J. A. M. Impactos antrópicos no regime hidrológico de tributários do rio Tocantins. 2017. $128 \mathrm{f}$. Dissertação (Mestrado em Recursos Hídricos em Sistemas Agrícolas) - Universidade Federal de Lavras, Lavras, 2017.

SÁ, R. A. Simulação hidrológica e projeções usando o modelo Swat nas vazões mínimas do rio Riachão, MG. 2016. 52f. Dissertação (Mestrado em Produção Vegetal no Semiárido) - Universidade Estadual de Montes Claros, Janaúba, 2016.

SANTOS, B. V. C. D. dos; FONTES, A. S. Modelagem hidrológica de sistema hídrico complexo do semiárido baiano. Revista Eletrônica de Gestão e Tecnologias Ambientais (GESTA), Santos, v. 2, n. 1, p. 152-162, 2014. DOI: http://dx.doi.org/10.17565/gesta.v2i1.10090

SILVA, R. M.; MEDEIROS, I. C. Análise hidrossedimentológica em ambiente SIG usando o modelo SWAT. GeoFocus, n. 14, p. 211-231. 2014. 
SOARES, L. C.; VELÁSQUEZ, L. N. M. Estimativa da recarga aquífera na bacia do rio Riachão, Norte de Minas Gerais. Águas Subterrâneas, São Paulo, v. 27, n. 2, p. 104-120, 2013.

DOI: https://dx.doi.org/10.14295/ras.v27i2.27359

VIEIRA, N. P. A.; PEREIRA, S. B.; MARTINEZ, M. A.; SILVA, D. D. DA; SILVA, F. B. Estimativa da evaporação nos reservatórios de Sobradinho e Três Marias usando diferentes modelos. Engenharia Agrícola, Jaboticabal, v. 36, n. 3, p. 433-448, 2016. DOI: http://dx.doi.org/10.1590/1809-4430-

Eng.Agric.v36n3p433-448/2016

VILAYSANE, B.; TAKARAA, K.; LUOB, P.; AKKHARATHC, I.; DUAN, W. Hydrological stream flow modelling for calibration and uncertainty analysis using SWAT model in the Xedone river basin, Lao PDR. Procedia Environmental Sciences, v. 28 p. 380-390, 2015.

DOI: https://dx.doi.org/10.1016/j.proenv.2015.07.047

VIGIAK, O.; MALAGÓ, A.; BOURAOUI, F.; VANMAERKE, M.; OBREJA, F.; POESEN, J.; HABERSACK, H.; FEHÉR, J.; GROŠELJ, S. Modelling sediment fluxes in the Danube River Basin with SWAT. Science of the Total Environment, Amsterdam, v. 599600, p. 992-1012, 2017. DOI: https://dx.doi.org/10.1016/j.scitotenv.2017.04.236

VELOSO, G. A.; LEITE, M. E.; ALMEIDA, M. I. S. DE. Geotecnologias aplicadas ao monitoramento do uso do solo na bacia hidrográfica do Riachão, no Norte de Minas Gerais. Revista de Geografia, Recife, v. 28, n. 2, p. 165 184, 2011.

WADA, Y.; DE GRAAF, I. E. M.; VAN BEEK, L. P. H. Highresolution modeling of human and climate impacts on global water resources. Journal of Advances in Modeling Earth Systems, v. 8, p. 735-763, 2016. DOI: https://dx.doi.org/10.1002/2015MS000618

XU, Y. P.; ZHANG, X.; RAN, Q.; TIAN, Y. Impact of climate change on hydrology of upper reaches of Qiantang River Basin, East China. Journal Hydrology, Amsterdam, v. 483, p. 51-60, 2013. DOI: https://dx.doi.org/10.1016/j.jhydrol.2013.01.004 